Néo-traditionalisme, autochtonie et gestion des ressources forestières dans le Mandé

Cas des villages de Ouoronina et Ticko de la commune rurale de Bancoumana au Mali

\title{
Seydou Keïta
}

\section{(2) OpenEdition}

\section{Journals}

Édition électronique

URL : http://journals.openedition.org/anthropodev/469

DOI : 10.4000/anthropodev.469

ISSN : 2553-1719

Éditeur

APAD - Association pour l'anthropologie du changement social et du développement

Édition imprimée

Date de publication : 1 avril 2014

Pagination : 69-99

ISBN : 979-10-93476-00-1

ISSN : 2276-2019

\section{Référence électronique}

Seydou Keïta, « Néo-traditionalisme, autochtonie et gestion des ressources forestières dans le Mandé

», Anthropologie \& développement [En ligne], 37-38-39 | 2014, mis en ligne le 01 décembre 2016, consulté le 06 mai 2019. URL : http://journals.openedition.org/anthropodev/469 ; DOI : 10.4000/ anthropodev.469

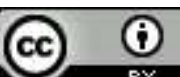

La revue Anthropologie \& développement est mise à disposition selon les termes de la Licence Creative Commons Attribution 4.0 International. 


\title{
Néo-traditionalisme, autochtonie et gestion des ressources forestières dans le Mandé
}

Cas des villages de Ouoronina et Ticko

de la commune rurale de Bancoumana au Mali

Seydou Keïta

\begin{abstract}
A travers les études de cas de deux villages voisins, cet article montre comment les populations autochtones se sont réappropriées la gestion des ressources naturelles en s'appuyant sur les institutions traditionnelles. Dans le contexte particulier de la décentralisation mais sans délégation officielle de gestion par l'Etat, le contrôle des ressources naturelles est devenu un enjeu majeur par rapport auquel les lignages dominants sont parvenus à conserver leur pouvoir face à la société civile.

Through case studies of two neighboring villages, this article shows how indigenous people recaptured the management of natural resources based on traditional institutions. In the particular context of decentralization without official delegation of management by the state, control of natural resources has become a major issue in relation to which the dominant lineages are able to maintain their power against civil society.
\end{abstract}

\section{Introduction}

La période précoloniale au Mali est marquée fondamentalement par une gestion autochtone des ressources forestières. Cette gestion sera perturbée par l'accaparement, dans les principes et dans les faits, des droits des populations par l'Etat colonial qui devient, à la fois, le propriétaire et le gestionnaire des ressources forestières, à l'exclusion de certains droits d'usage reconnus. La progression de l'économie marchande au détriment d'une économie domestique extra-monétaire conditionnera de nouvelles formes d'appropriation et d'exploitation de ces ressources. Cette situation n'a pas été démentie jusque dans un passé récent par l'Etat indépendant. 
La politique de la décentralisation au Mali1, énoncée en 1993 et mise en actes au niveau rural à travers les élections communales de mai 1999, reconnaît aux collectivités décentralisées leur souveraineté sur les ressources forestières.

La révision des textes forestiers en 1995 marque une rupture formelle avec le caractère unilatéral des décisions de l'Etat sur les ressources naturelles. Il s'agit de la promulgation d'une série de lois et décrets qui se sont substitués au code forestier de 1986. Les lois sont relatives à l'exploitation, au commerce et au transport du bois. Elles fixent les conditions de la gestion des ressources forestières et traitent de la constitution et de la gestion du domaine des collectivités territoriales. Les décrets fixent, quant à eux, les taux et les modalités de recouvrement et de répartition des taxes perçues à l'occasion de l'exploitation du bois dans le domaine forestier de l'Etat. L'application de ces mesures édictées reste encore à l'épreuve de l'évaluation. Dans le même temps, on constate la montée des revendications communautaires par rapport à la gestion des ressources. Le présent article rend compte d'un exemple de gestion basé sur la réappropriation de certaines valeurs et pratiques du passé par les communautés villageoises, derrière une forme de néo traditionalisme et une (re)définition de l'autochtonie, dans deux villages de la commune rurale de Bancoumana, ainsi que des conséquences qui en découlent sur le plan de la gouvernance locale et de l'accès à la ressource.

1 Cf. Présidence de la République du Mali, Mission de la Décentralisation et des Réformes Institutionnelles, 1999. Lois et Décrets sur la décentralisation, Bamako, 4ème édition. 


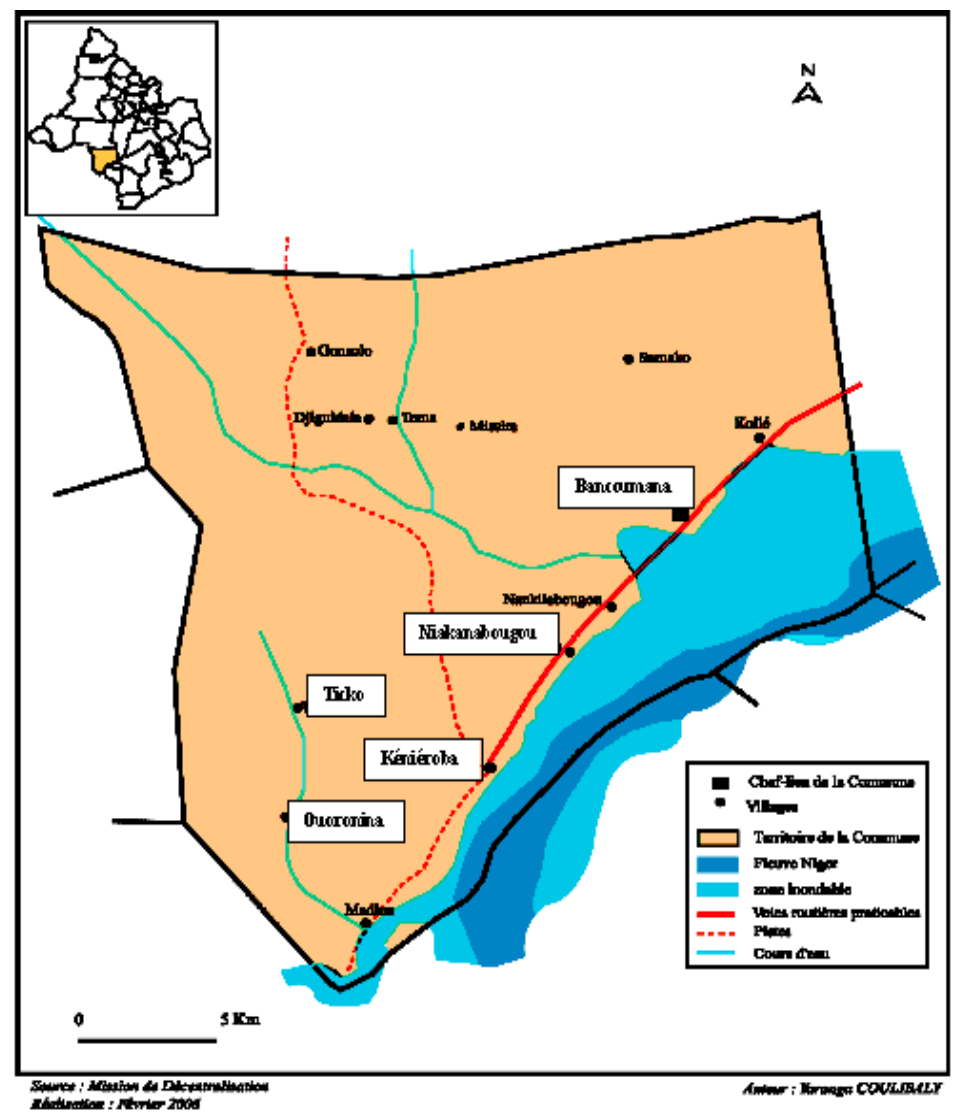

Carte 1 : La commune rurale de Bancoumana

\section{La commune rurale de Bancoumana}

\section{Présentation}

La commune rurale de Bancoumana est située à l'ouest de la zone du Mandé, à $60 \mathrm{~km}$ de Bamako. D'après le document du Programme de développement économique social et culturel (PDSEC, 2004-2009) de la com- 
mune, elle compte 14 villages et regroupe environ 22000 habitants dont $90 \%$ appartiennent à l'ethnie malinké, les 10\% restant étant composés de plusieurs autres ethnies minoritaires.

\section{Les forêts de Bancoumana}

Les forêts couvrant la commune sont constituées en deux grands massifs comprenant des forêts naturelles non classées. Le premier est situé dans un triangle qui s'étend sur une superficie de $60 \mathrm{~km}^{2}$ et se situe aux abords des villages de Madina à l'ouest, Kéniéroba, Niaganabougou, Nanguilabougou à l'ouest, Téma, Djiguidala et Niamé au nord. Les villages de Ouoronina et Ticko sont à l'intérieur de ce massif qui se prolonge dans la commune voisine de Karan, au sud-ouest. Il est constitué d'une savane arbustive et arborée ainsi que de forêts galeries le long des cours d'eau. Le second massif est situé dans une dépression entre le fleuve et le plateau. II est le plus dense mais constitué, surtout, d'arbres aquatiques dont l'exploitation ligneuse n'est pas perceptible. Chaque village dispose de son espace forestier conformément aux droits coutumiers de possession des terres.

Sur le plan de la tradition, la forêt en tant que telle ne constitue pas une entité à part. Elle est toujours liée à la terre et prise en compte avec elle. Dans la conception de l'ethnie malinké de cette partie du Mandé, la terre importe plus que ce qu'elle porte. De plus, comme dans beaucoup d'autres sociétés africaines, si les terres appartiennent à des lignages, en revanche, aucun lignage ne peut se prévaloir en particulier de la propriété des ressources forestières qu'elles recèlent. Ces ressources sont classées parmi les biens d'intérêt collectif (Sawadogo, 2001 : 107) pour lesquels la jouissance des droits, la protection et la responsabilité de gestion sont égales pour tous.

Jusqu'à l'avènement de la colonisation, la forêt n'avait pas de valeur marchande, elle était accessible à toute la communauté villageoise pour la jouissance des droits d'usage. Alors que l'exploitation de la terre était sujette à des démarches auprès des chefs coutumiers, l'exploitation forestière, elle, était libre dans la limite de la satisfaction des besoins domestiques. Toute la population, sans distinction de lignage, pouvait profiter des produits issus de la forêt sans aucune pression ni revendication. Cependant, le déboisement complet d'une terre dont on n'était pas proprié- 
taire n'était pas autorisé car cela équivalait à une revendication de l'espace concerné.

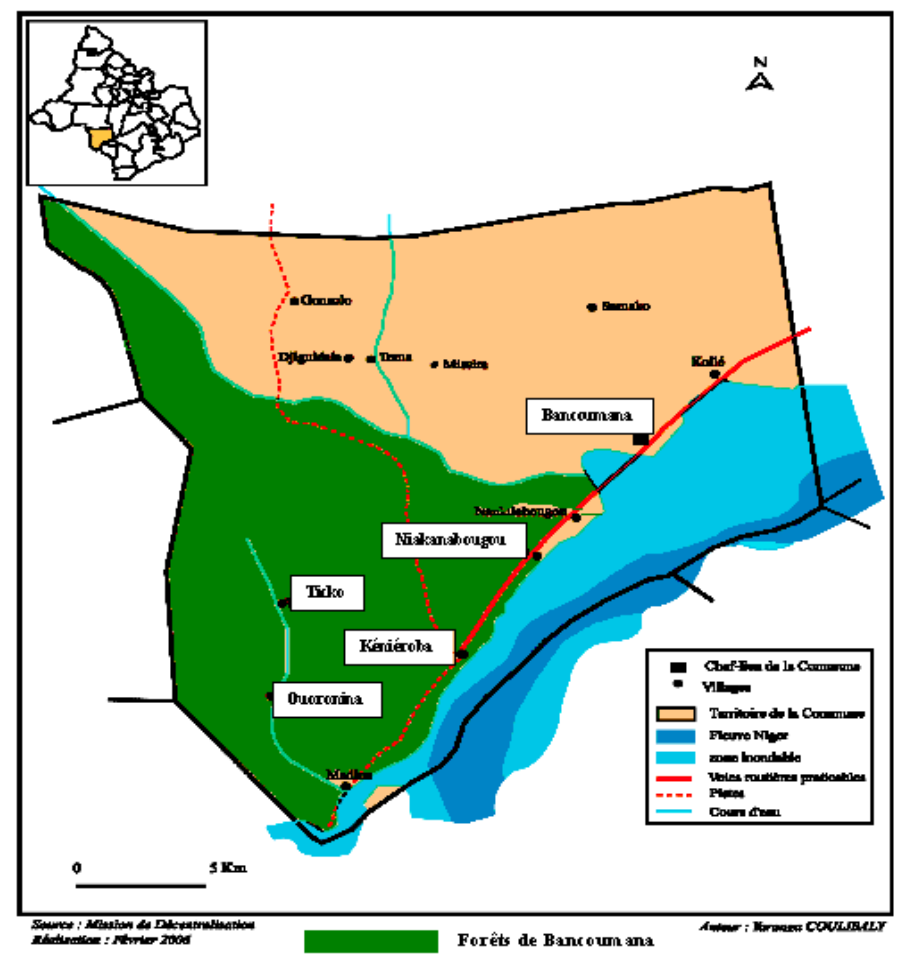

Carte 2 : Forêts de Bancoumana

Ce modèle de gestion, basé sur les valeurs ancestrales, a été mis à l'épreuve avec la marchandisation des ressources. Ainsi, on peut désormais constater une sorte de néo-traditionalisme dans la gestion, qui s'appuie sur la définition de l'autochtonie et où s'affirme un discours de revendication communautariste de la forêt villageoise ainsi que certaines formes d'ostracisme ou d'exclusion à l'égard des " étrangers " dans l'exploitation des ressources. C'est cette situation qui prévaut dans les villages de Ouoronina et Ticko. 
Organisation socio-traditionnelle autour de la gestion des ressources forestières à Ouoronina et Ticko

\section{Le village de Ouoronina}

Peuplé d'un millier d'habitants environ ( $d$ 'après le recensement général de la population et de l'habitat de 1998), Ouoronina est situé au bord de la rivière du même nom, au sud de la commune de Bancoumana et à $7 \mathrm{~km}$ de la route principale qui mène à Kangaba. Fondé par un chasseur appartenant à un lignage patrilinéaire du clan Keïta, précédemment installé à Djongoya-Ngouagnan, le village est implanté sur les terres des Camara du village voisin de Ticko. D'autre lignages s'y sont par la suite installés tels que ceux des Camara, Cissé et Traoré. La chefferie est patriarcale et gérontocratique. Seuls des membres du lignage Keïta se succèdent à la tête du village, tirant cette légitimité de leur descendance vraie ou supposée de Soundiata Keïta, le fondateur de l'empire du Mali au $13^{\text {ème }}$ siècle.

L'activité économique repose principalement sur l'agriculture et l'orpaillage traditionnel, qui rapporte quelques revenus supplémentaires pendant la saison sèche. On constate aussi la migration des jeunes vers Bamako, la Guinée et même l'Espagne. Les activités forestières sont secondaires dans le système de production mais les revenus qu'elles génèrent permettent à un certain nombre de ménages de faire face aux besoins monétaires.

\section{La forêt de Ouoronina}

La forêt est située dans le triangle formé par les villages de Karan, Tiko et Ouoronina. Elle semble être bien conservée vu les ressources abondantes qui s'y trouvent. Les arbres fruitiers tels que le néré (Parkia biglobosa) et le karité (Butyrospermum parki) font l'objet d'une protection particulière. La forêt procure aux villageois un ensemble de produits. L'exploitation du bois de chauffe rapporte des revenus à ceux ou celles qui s'adonnent à cette pratique. Pendant la saison sèche, les femmes ramassent du bois mort dont une partie est utilisée directement pour la cuisson des aliments et l'autre est destinée à la vente. Elles pratiquent aussi la coupe du bois vert qui est stocké pour les besoins de chauffage pendant la saison hivernale. Le bois d'œuvre est utilisé dans la charpenterie au sein du 
village (toiture des maisons, hangars pour s'abriter du soleil). Les arbres les plus gros servent pour la menuiserie - bois pour la confection des meubles. Leur abattage est rigoureusement contrôlé par la chefferie du village. Les essences comme le caïlcédrat (Kaya senegalensis Juss), le "lengue » (Afzelia africana Smith) et le " ngalama " (Anogeisus leiocarpus), bien que protégées (article 17 de la loi 95 -004), sont très convoitées et constituent une source de conflits opposant parfois les exploitants de bois venant de Bamako et de Kangaba à la chefferie du village. Enfin, certaines essences forestières servent de fourrage foliaire au bétail et les racines, écorces et feuilles de certains arbres et arbustes sont utilisées comme médicaments dans la pharmacopée traditionnelle.

L'état de la ressource paraît satisfaisant jusqu'à présent. Toutes les espèces et produits forestiers dont les populations locales ont besoin s'y trouvent. Cependant, on remarque une grande quantité d'arbres morts, imputable non seulement à l'insuffisance hydrique des sols suite à une pluviométrie déficitaire d'année en année mais aussi aux feux de brousse qui sont très fréquents après chaque saison pluvieuse.

\section{Appropriation et contrôle de l'accès à la forêt}

La loi 95 - 004 (article 24) définit trois domaines forestiers : ceux de l'Etat, des collectivités territoriales et des particuliers. Ces trois domaines constituent le domaine forestier national. L'exploitation et les droits d'usage sont aussi définis par la même loi. En principe, la forêt de Ouoronina fait partie du domaine forestier des collectivités territoriales et sa gestion devrait donc relever des autorités communales de Bancoumana. Mais, aucun transfert de gestion de domaine forestier n'a jusqu'ici été effectué au profit de la commune. Cependant, dans le secteur de Ouoronina, un massif forestier a fait l'objet d'une délimitation et d'un aménagement technique dans le cadre du projet Stratégie Energie Domestique (SED) mis en oeuvre en 2000. Cela a été réalisé contre l'avis de la population et au profit du seul hameau de Nialafoura qui relève normalement du village de Ouoronina.

On remarque globalement que la réglementation forestière est insuffisamment appliquée dans le secteur. La structure de contrôle de l'Etat, à savoir l'antenne du Service de la conservation de la nature, dispose de très peu d'agents (trois agents forestiers) pour couvrir un territoire qui s'étend 
sur quatre communes. L'agent forestier affecté à la commune de Bancoumana aurait de plus été congédié par les populations qui ont systématiquement refusé de se soumettre à son contrôle. Cette situation dénote de l'incapacité de l'Etat à faire appliquer ses propres règles. II y a une appropriation de fait des ressources forestières par la chefferie villageoise qui en détermine les droits d'accès et le contrôle. La superposition des droits qui résulte de cette appropriation montre une affirmation des droits coutumiers qui repose sur une organisation sociale structurant la gestion des ressources. Cette organisation tire sa cohérence d'une structure sociale basée sur la cohabitation des clans Keïta, Traoré, Cissé, Camara et des lignages correspondants. Ces clans sont représentés dans le conseil de village qui est l'instance du pouvoir au niveau local. Comme nous l'avons $\mathrm{vu}$, le chef de village est toujours issu du kabila (clan) Keïta, de par la légitimité historique qu'il se donne à travers Soundiata mais aussi de par I'histoire de la fondation du village dont un chasseur Keïta serait à l'origine. Cependant, ce dernier aurait en fait demandé des terres aux Camara de Ticko pour s'y installer. Si le pouvoir appartient donc aux Keïta, ces derniers reconnaissent tout de même que les membres du clan Camara sont propriétaires des terres. Le conseil de village ne se tient jamais sans la présence d'un de leurs représentants.

La forêt est, elle, un espace approprié appelé kungo da qui appartient à la communauté villageoise. Chaque membre de la communauté reconnaît son kungo da et ses limites par rapport aux autres. La gestion de la forêt revient à trois organisations principales du village, à savoir le conseil de village (dugutigi bulon sigui), les kari (l'organisation des classes d'âge) et le donso ton (association des chasseurs). Voyons maintenant comment les ressources forestières sont gérées dans la pratique.

\section{Le Comité Villageois de Gestion des Ressources Naturelles (CVGRN) : une greffe qui n'a pas pris}

Créé dans le cadre étatique du Projet de Gestion des Ressources Naturelles (1992-2000), le CVGRN était une organisation villageoise qui devait, en principe, contribuer à renforcer les capacités de gestion des habitants en leur transmettant les compétences nécessaires pour assurer une bonne gestion des ressources naturelles et de leur terroir. II s'agissait de restituer aux populations le pouvoir de gestion, sur le principe de la participation de 
toutes les catégories socioprofessionnelles impliquées dans l'utilisation des ressources forestières, fauniques, halieutiques, hydrauliques et pastorales. Le CVGRN devait travailler selon un plan d'aménagement et de gestion établi par les techniciens du projet. La protection de la zone forestière revenait aux villageois par la mise en place de pare-feux dans l'objectif $d^{\prime}$ 'une meilleure exploitation. Mais, faut-il le souligner, le modèle était loin d'être endogène et autonome. II demandait une participation financière ou en nature des populations dans les activités initiées et celles-ci sont restées confuses face aux mécanismes administratifs mis en place. Les concepts tels que ceux de cofinancement, de convention, de passation de marchés, d'adjudication, de gestion de contrats leur étaient totalement étrangers et elles n'ont pas su s'approprier de ce mode d'organisation.

Le CVGRN de Ouoronina, parachuté sur les populations, n'a donc pas fonctionné. Les populations ne se sont pas reconnues dans le dispositif institutionnel mis en place, malgré une reconnaissance formelle du CVGRN au niveau de l'administration. C'est pourquoi, à la fin du projet, en 2000, le pouvoir coutumier a eu tôt fait de récupérer sa légitimité de gestion en s'appuyant sur des institutions néo-traditionnelles symbolisées par le kari et le donso ton par un retour endogène aux traditions, basé sur les expériences du passé.

\section{Le conseil de village, les "kari" et le "donso ton"}

Si le conseil de village peut être considéré comme l'organe de décision de la gestion coutumière des ressources, les kari et le donso ton en constituent les organes d'exécution.

Selon la tradition, les kari sont des garçons d'un même groupe d'âge ayant subi ensemble l'épreuve de la circoncision et les activités d'initiation qui y sont liées. "Les circoncis et les excisées qui ont subi l'opération dans le même intervalle de sept ans, forment ce que les Malinké appellent un kari » (Leynaud et Cissé, 1968 : 313). Le kari est un groupe structuré qui reçoit un nom ${ }^{2}$, a ses cadres et ses règles de conduite. Les kari forment les

2 «Les noms donnés aux promotions de circoncis évoquent soit des événements météorologiques et astronomiques, soit des qualités fondamentales pour l'homme, soit des noms d'animaux mythiques et "totémiques" » (Leynaud \& Cissé, 1968 : 313-314). 
sociétés de travail, d'entraide et de loisirs dans les villages et concernent toutes les générations ainsi qu'on en retrouve plusieurs dans le même village. Ceux des aînés dirigent les activités et les plus jeunes exécutent les tâches qui leur sont assignées, jusqu'à l'avènement d'une nouvelle promotion.

C'est ainsi que, par le passé, les jeunes gens parcouraient la forêt dans tous les sens pour forger leur endurance personnelle, s'initier à la reconnaissance des différentes essences forestières et des symboles rattachés aux espèces végétales, à la topographie et à la toponymie des lieux. Les rites initiatiques étaient pratiqués dans les bois sacrés tout autour du village. Les cérémonies rituelles, notamment les sacrifices ou offrandes (appelées tambacoudoun) faites aux arbres étaient accomplies par les kari. II s'agissait de sacrifier un taureau blanc et de faire des libations en vue de solliciter la faveur des génies de la brousse pour assurer de bonnes récoltes et des évènements heureux pour le village. Aucun kari, après la circoncision de ses membres, ne pouvait prendre possession de la place publique du village sans accomplir le rite de l'offrande à l'arbre. Chaque génération de kari devait aussi, pendant quatre ans, se rendre maître de la place publique du village avant de passer le relais à la génération suivante. Enfin, la brousse était considérée comme un lieu sacré qu'il fallait protéger et conserver. L'ensemble de cette tradition est tombée en désuétude à cause de l'influence de l'islam et du fait que les bois sacrés et les gros arbres autour desquels se déroulaient ces cérémonies ont tous disparu.

De nos jours, les ton (associations), dans les villages malinké, sont constitués d'après les kari. Ces derniers sont aussi toujours largement impliqués dans la surveillance de la brousse, selon des principes qui leur sont propres. Les jeunes les plus actifs forment des groupes de surveillance qui luttent contre les feux de brousse et la coupe du bois vert. Ils se chargent aussi de la vérification des permis délivrés par l'administration forestière et des quotas alloués, ainsi que l'application stricte des consignes d'exploitation données par le conseil de village. Les personnes suspectées de fraude subissent un interrogatoire et les récalcitrants sont conduits devant les chasseurs (donsow). Ces groupes, en tandem avec l'association des chasseurs (donso ton), jouent donc le rôle de police forestière, l'Etat n'ayant pas les moyens d'assurer ce rôle au niveau du village. 
Le donso ton est l'association traditionnelle des chasseurs du village. II est aussi organisé selon les principes des kari. Les chasseurs se divisent en deux groupes : les donso ou "initiés", qui ont reçu les leçons du dankun (point de croisement des chemins en dehors du village, où se déroulent les cérémonies initiatiques) et les " non initiés ", qui savent tirer au fusil pour abattre le gibier mais qui ne sont jamais passé par le dankun. Ces derniers sont appelés nantan (chasseurs non initiés). Les donso et les nantan se répartissent les différents points de surveillance dans la forêt. Les piquets de surveillance, définis pour chaque catégorie de chasseurs, durent trois mois. Signalons ici que les différents groupes de chasseurs sont aussi organisés en brigades de surveillance sous la supervision du chef tutélaire, le donso ba. Nous assistons en fait à un véritable quadrillage de la forêt et à sa mise en défens.

Cette forme d'organisation, à la fois éducative, politique et économique au niveau du village, s'est en quelque sorte superposée à l'Etat pour mener une gestion de proximité des ressources forestières au profit des populations.

\section{Les pratiques actuelles de contrôle des ressources}

Dans le contexte des dispositifs étatiques des projets de gestion participative des ressources forestières que nous avons mentionné ci-dessus, les massifs forestiers de la région ont été classés selon leur type d'exploitation et les aménagements réalisés. Ainsi, certains massifs faisaient l'objet d'une exploitation " contrôlée ", " orientée " ou bien " incontrôlée ». A chaque type correspondait une forme d'organisation de contrôle plus ou moins contraignante et réglementée ${ }^{3}$.

Dans notre cas, l'exploitation forestière est de type incontrôlé, c'est-àdire qu'elle concerne un massif forestier non délimité et non aménagé (loi 95 -003). Les services forestiers délivrent alors des permis aux exploitants sans discrimination. Cependant, dans les limites du village, les droits d'usage sont réservés aux seuls résidents. Les étrangers - même munis de permis d'exploitation -, doivent s'adresser au chef de village qui leur donne l'autorisation d'accéder à la ressource. Ils doivent aussi spécifier la qualité

3 Cf. le texte de F. Mbodj supra dans ce même numéro. 
et la quantité de la ressource qu'ils entendent exploiter. Les chasseurs sont chargés de contrôler leur accès à la ressource. Ils doivent indiquer aux exploitants les lieux où le produit demandé est disponible et veiller au respect de la quantité à prélever. Ils vérifient, d'une part, la quantité et la qualité du produit avant le transport et veillent, d'autre part, à ce qu'aucun exploitant, qu'il soit autochtone ou étranger, ne dépasse son quota. Ensuite, les exploitants payent aux autorités traditionnelles des redevances forfaitaires qui peuvent atteindre 25000 FCFA par exploitant. Cette taxation est illégale au regard des textes forestiers mais légitime pour le pouvoir coutumier.

Les contrevenants aux règles établies sont traqués et arrêtés par les chasseurs et traduits devant le conseil de village qui décide de leur verbalisation. Cela a été le cas en mars 2006, lorsque des exploitants étrangers venus de Kangaba s'étaient infiltrés dans la forêt pour abattre de gros arbres sans l'autorisation du conseil de village. Au nombre de cinq, ils ont été arrêtés en brousse par les piquets de surveillance de l'association des chasseurs et le conseil de village leur a infligé une amende de 30000 FCFA. La somme a été versée à la caisse du village plutôt qu'aux forestiers. Pour fixer cette taxation, le conseil de village s'est inspiré de l'ensemble des charges communautaires qui lui reviennent (paiement des salaires des enseignants et de l'infirmier, réception des officiels, etc...) et a veillé à ne pas dépasser les normes de l'Etat en la matière. II n'y a cependant pas de barème de taxation écrit pour les infractions commises. Tout se gère au sein du dugutigui blon.

Une telle situation est fréquente et peut concerner aussi les pasteurs transhumants. II existe en effet de nombreux cas de conflits avec les éleveurs qui viennent souvent de très loin, des zones de Banamba, de Bamako et même de Mauritanie. Au sein du conseil de village, les ressources naturelles sont considérées comme la propriété exclusive du village. Selon B.K., un proche du chef de village :

"Les transhumants doivent s'en tenir aux règles fixant la rentrée et la sortie des animaux des zones pastorales. Nous ne devrions même pas les recevoir mais ils ont droit à la ressource, comme nous. Ce qui fait problème entre nous, c'est que nos troupeaux se nourrissent $d^{\prime}$ herbe pendant que ceux des éleveurs transhumants se nourrissent de feuillage. Donc ils contribuent à la dégradation de notre couvert 
végétal. C'est cette situation qui les oppose à nous. Quand des éleveurs nous arrivent avec des haches, nous devrions prendre des dispositions ».

Le chef de l'association des chasseurs raconte aussi :

"Le rôle de notre association, c'est de garder la brousse pour empêcher les dégâts qui pourraient être causés dans la forêt. C'est notre espace vital, nous devrions le protéger. Si nous arrêtons un éleveur en train de couper une branche d'arbre, il doit payer 5000 FCFA pour le compte du village. En cas de résistance, nous confisquons l'une de ses bêtes jusqu'à ce qu'il s'exécute ".

Ces propos montrent que cette association semble bien jouer son rôle de protection de la forêt.

Nous avons observé que les populations, à travers ces organisations néo-traditionnelles (elles prennent leur essence à la fois dans les organisations traditionnelles et les CVGRN), assurent un véritable contrôle sur les ressources forestières et montrent une certaine efficacité de gestion dans le sens de la conservation des ressources. Le rôle de plus en plus accru des chasseurs dans la gestion environnementale s'explique par les carences de l'Etat en matière d'organisation sociale pour la gestion des ressources naturelles.

Nous constatons aussi qu'il y a un accès libre aux ressources, excepté quelques réticences par rapport aux comportements des populations dites étrangères vis-à-vis de ces ressources. A titre d'exemple, les troupeaux des villageois ont un droit de parcours sur toute l'étendue du kungo da et même dans celui des villages voisins, étant donné qu'il s'agit d'une même communauté ethnique malinké dont les clans et lignages entretiennent depuis des périodes anciennes des relations de parenté et d'alliance. Tel n'est pas le cas pour les pasteurs transhumants qui négocient les droits d'accès au cas par cas. Chaque groupe de pasteurs doit se trouver un logeur (diatigi) dans le village s'il veut pouvoir accéder au territoire forestier. En contre partie, ce groupe doit contribuer à la fertilisation des champs de son logeur en pratiquant le parcage de son bétail dans ces champs. II s'instaure aussi un système d'échanges selon lequel les pasteurs se procurent des céréales auprès des villageois et ceux-ci, en retour, peuvent acheter des bœufs à un prix raisonnable. Très rarement, il existe des alliances 
matrimoniales entre ces communautés de pasteurs appartenant généralement à l'ethnie peulh et les agriculteurs de l'ethnie malinké. La coopération oscille entre la complémentarité économique et la concurrence dans l'accès aux ressources naturelles et aux espaces. Le même phénomène a été constaté dans le nord de la Côte d'Ivoire, entre les éleveurs peulh et les paysans sénoufo :

"L'importance accordée aux bovins dans la société sénoufo contemporaine tient à des motifs purement économiques qui poussent de plus en plus de paysans à faire des placements de bœufs. L'obligation de recourir aux peulh, doués de compétences dans la gestion des bovins, fonde la division ethnique du travail entre ceuxci et les sénoufo. Le salaire, la consommation et la commercialisation du lait sont les principaux avantages que les bouviers peuls tirent de leur service chez les sénoufo " (Diallo, 1995 : 3-4).

Les relations deviennent conflictuelles quand le bétail des transhumants cause des dégâts dans les champs de culture.

\section{L'exploitation du bois : une activité informelle génératrice de revenu}

Dans le village de Ouoronina, chaque villageois sait que la forêt constitue une rente pour lui. Pendant la saison sèche, le bois est majoritairement exploité par les femmes. Chaque exploitant ou exploitante autochtone du village se fournit en bois dans la forêt en accès libre. Sauf cas de fraude, c'est le bois mort qui est ramassé. Le charbonnage et l'utilisation de tronçonneuses pour abattre les arbres sont des actions strictement interdites. Un marché de bois informel est installé au village où les commerçants transporteurs viennent acheter du bois de chauffe. Selon nos propres données d'enquête, le fagot est vendu à 50 FCFA. La recette de chaque exploitant (dont le nombre est estimé à 75) pendant la période d'exploitation, c'est-à-dire de novembre à avril, s'élève en moyenne à 50000 FCFA, soit 1000 fagots par exploitant. Cela représente une somme totale de 3500000 FCFA, uniquement obtenue par la vente du bois mort, ce qui démontre que les ressources forestières jouent un rôle important dans la constitution des revenus des villageois. En cas de déficit céréalier, il a aussi été constaté une recrudescence de l'exploitation du bois pour faire face aux besoins domestiques. "Pendant les années de disette, l'autorisation 
est donnée à chaque villageois par le conseil de village pour couper le bois vert, afin de trouver à manger aux enfants " disait M.K., un autochtone du village.

Très rarement les services forestiers de l'Etat viennent pour des contrôles au village. En revanche, ils sont saisis par les villageois en cas de litige avec les communautés voisines ou lorsqu'ils veulent faire des défrichements pour avoir de nouvelles terres agricoles. Dans ce cas, ils doivent obtenir des autorisations auprès du service forestier, sans que cela n'élimine, toutefois, le contrôle communautaire qui s'exerce sur les ressources. Celui-ci repose sur les décisions collectives quant à la surveillance des ressources mais aussi sur la répression qui peut être exercée à l'encontre les fraudeurs.

\section{Quand une communauté voisine particulière "fait feu de tout} bois»

La convoitise des communautés voisines sur les ressources a provoqué une prise de conscience quant à l'implication de toute la communauté dans leur gestion. II est intéressant ici de signaler la présence, dans le secteur, d'un camp pénal pour lequel l'Etat a autorisé le bûcheronnage aux prisonniers dans le cadre de la rééducation des repris de justice et de leur réinsertion socio-économique. Pour cela, les prisonniers ont été associés à l'exploitation du massif forestier de Nialafara, un hameau de Ouoronina, délimité dans le cadre de l'implantation des marchés ruraux de bois. Là, l'exploitation est de type orienté et placée sous le contrôle de la structure rurale de gestion (SRG). II faut retenir que les détenus, du fait de leur statut, ne sont pas associés à la gestion. Ils sont considérés comme de simples exploitants sans droit d'éligibilité au bureau de la structure rurale de gestion.

Les entretiens menés auprès des membres du bureau de la SRG de Nialafara nous évoquent naturellement une forme de méfiance envers les détenus. Ils sont accusés, pour l'occasion, de ne pas respecter les principes de la gestion rationnelle (règles autour de la coupe des arbres, respect des quotas, vente des produits sur le marché rural de bois, etc.) instaurée au sein du massif délimité et, encore moins, dans la zone d'exploitation incontrôlée de Ouoronina. II leur est reproché de s'adonner à la coupe abusive du bois (les détenus ne respecteraient pas les quotas fixés) dans le 
domaine de Ouoronina pour en faire du charbon alors que cette activité est interdite par le conseil de village. Les autorités villageoises ne peuvent cependant pas s'y opposer dans la mesure où ce sont les services de l'Etat qui les autorisent, mais elles souhaitent que cela ne se fasse pas au détriment de la sauvegarde de leurs ressources forestières. "Si l'Etat pouvait nous débarrasser de ce voisinage encombrant, ce serait une bonne chose " disait le chef de village. Cependant les autorités villageoises demeurent impuissantes face au nombre de prisonniers du camp pénal de Kéniéroba qui détiennent l'autorisation d'exploiter de la part de l'Etat. En revanche, les autochtones leur reprochent des pratiques qu'ils considèrent comme illégales, telles que la coupe du bois vert et le charbonnage.

Les prisonniers représentent une catégorie particulière d'étrangers. S'il y a une relation de complémentarité sur certains plans entre les transhumants et les villageois, comme nous l'avons montré plus haut, il n'en est pas de même avec les détenus. Bien qu'étant dans les liens de la détention, ces derniers se sentent indépendants des villageois et bénéficient d'une certaine manière de la couverture de l'Etat. Ils ne se voient pas dans l'obligation de respecter les règles de gestion établies par les autorités villageoises et semblent être à l'abri des sanctions sociales qui peuvent frapper les autres catégories d'exploitants. Cela pourrait expliquer leur comportement par rapport à la gestion des ressources. Par ailleurs, les autochtones tirent peu d'intérêt de leur présence, c'est pourquoi ces détenus peuvent être accusés de tous les maux et, souvent, sont désignés comme coupables en lieu et place de certains autochtones aux mauvais agissements. II n'y a en fait pas de certitude sur tout ce qu'on leur reproche. Cela rentre dans le cadre de la stigmatisation de l'étranger, surtout quand celui-ci ne partage pas ses compétences avec l'autochtone. Enfin, d'après les renseignements que nous avons recueillis, les populations du secteur de Ouoronina ne connaissaient pas la technique du charbonnage : elle aurait été introduite par les détenus. Ainsi, on peut comprendre, dans une certaine mesure l'interdiction du charbonnage par les autorités villageoises.

On voit donc là qu'il y a une compétition autour de la ressource entre les autochtones et cette catégorie particulière d'étrangers que sont les détenus. Ces derniers " ont fait feux de tout bois " en cherchant à tirer le meilleur parti de cette compétition, c'est à dire en exploitant le maximum 
de ressources pour satisfaire des besoins que l'Etat ne peut assurer. II faudrait ajouter que les détenus ne bénéficient pas des droits d'usage au même titre que les autochtones à cause de leur statut. On comprend dès lors qu'il y a une volonté des autochtones à conserver les ressources à leur seul profit en essayant de montrer la figure de l'étranger comme destructrice de la ressource.

\section{Le village de Ticko}

Peuplé de 571 habitants, le village de Ticko est distant de $7 \mathrm{~km}$ de Ouoronina. La spécificité de ce village apparaît dans le pouvoir mystique attribué au clan Camara et à ses différents lignages qui peuplent le village. Camara (2002: 46) rapporte que tout ce pays appartenait au roi de Ticko, dont les terres s'étendaient jusqu'à Kolen, Karan, Deguela, Gonsolo, Selenkenyin et Nyamèn (l'ensemble de la commune de Bancoumana et de celle, voisine, de Karan). L'autochtonie des Camara est fortement affirmée dans les traditions de toute la région. Leur ancêtre mythique du nom de Ticko Jamba, mi-humain, mi-animal, serait descendu du ciel par une chaîne et se serait rendu maître de toutes les terres. "Les origines des autres clans sont connues, mais en ce qui nous concerne, les Camara de Ticko, nos origines sont directement divines. "n'y a aucun mélange parmi nous.", disait le chef de village. Contrairement à beaucoup de villages du Mandé dont les chefferies sont représentées par le clan des Keïta, à Ticko ce sont donc les Camara qui assurent le commandement. Cette suprématie des Camara sur les autres clans Keïta, Traoré et Soukané du village se traduit dans la réalité à travers leur pouvoir de gérer les ressources naturelles.

A Ticko, toutes les ressources naturelles (terres, forêts, eaux) appartiennent aux Camara. Ce sont eux qui détiennent tous les droits d'accès. Ici, les ressources du kungo da sont très abondantes et convoitées et il existe un dispositif de gestion des ressources forestières à l'image de celui de Ouoronina. "Personne ne peut se hasarder avec une hache ou une tronçonneuse dans notre brousse sans notre autorisation " aime à répéter le chef de village. La surveillance de la forêt revient à chacun des villageois (hommes, femmes, enfants). Mais le rôle de coercition appartient au donso ton (l'association des chasseurs).

En 2006, certaines personnes se disant ressortissantes du village voisin de Kéniéroba sont entrées dans le kungo da de Ticko avec une tronçon- 
neuse et sans aucune autorisation pour abattre certains gros arbres. Mal leur en pris. Elles ont été arrêtées par les chasseurs et conduites devant le conseil de village qui leur a infligé une lourde amende de 50000 FCFA et leur a confisqué le bois. A la question de savoir comment le conseil de village avait pu appliquer cette sanction, l'un des conseillers du village a répondu : " notre pouvoir mystique, car nous sommes capables de neutraliser nos ennemis par ce pouvoir. Tous les gens qui nous connaissent ont peur de nous à cause de ce pouvoir ». Ce pouvoir est reconnu par toutes les communautés de la contrée et même au-delà.

La forêt de Ticko semble être gérée avec parcimonie. Les prélèvements effectués par les villageois eux-mêmes se limitent strictement aux besoins domestiques (bois de chauffe, bois d'œuvre, bois de service). L'exploitation marchande du bois y est interdite. Les artisans du bois pratiquent leur métier uniquement pour la satisfaction des besoins des villageois. Par exemple, si un villageois a besoin de madriers pour la charpente de sa maison, il fait le prélèvement nécessaire de bois et passe un contrat avec le menuisier qui vient faire le travail sur place, au village. Ce dernier est toujours rémunéré en espèce, jamais en nature (bois). Le travail de scierie est par ailleurs interdit en forêt. Les villages voisins de Kéniéroba et de Niamey sont aussi fournis en bois de construction sur présentation, par les postulants, de dix noix de cola au chef de village en guise de demande d'autorisation. A ce moment, un des chasseurs est désigné pour leur montrer dans la forêt l'emplacement du produit demandé. Les demandeurs ne peuvent en aucune façon dépasser le quota qui leur a été octroyé. En cas de dépassement, le reliquat est restitué au conseil du village qui procède au partage entre les villageois qui en ont besoin.

Aucune compensation n'est demandée aux ressortissants des villages cités du fait de leurs liens de parenté et parce qu'ils ne bénéficient pas tous de l'accès à des ressources forestières exploitables en raison de l'inégale répartition de celles-ci sur le territoire. C'est le principe de la solidarité dans le partage des ressources. Cependant les pasteurs transhumants sont pour leur part totalement interdits d'accès à la forêt. Cela nous a été confirmé par un pasteur que nous avons rencontré dans la forêt de Ouoronina : 
"Nous sommes reçus dans tous les villages du Mandé sauf à Ticko. Nous contournons son espace forestier. Les autochtones des villages voisins ont peur d'eux, à plus forte raison nous, les étrangers ».

Nous voyons ici que la notion " d'étranger " s'intériorise dans la représentation des éleveurs transhumants face à la difficulté d'accès aux ressources naturelles et aux espaces de pâturage.

La gestion des ressources forestières à Ticko est basée sur les règles de gestion traditionnelle qui ne semblent pas être encore entamées par l'appât du gain propre à l'exploitation marchande. Le principe de conservation et de protection des arbres est sous-tendu par des croyances en des forces surnaturelles. Le chef de village nous a ainsi déclaré :

"Nous tenons nos pouvoirs occultes des génies qui sont logés en forêt. Donc nous ne pouvons accepter qu'en aucune manière, des personnes extérieures à notre village viennent détruire notre forêt. Cela équivaudrait à la destruction de nos pouvoirs occultes comme nous l'avons déjà constaté avec la disparition de certains de nos bois sacrés, sièges de l'accomplissement de nos rites et rituels. En plus de cela, notre village n'a jamais connu de sécheresse. L'abondance des arbres y est de beaucoup dans cette situation ".

Il apparaît ici que, dans la représentation des autorités traditionnelles, leur pouvoir de gestion sur les ressources permet de maintenir l'équilibre entre le naturel et le surnaturel, en tant que dimensions de l'univers qui ne sont aucunement dissociées. C'est le rapport à l'invisible qui leur permet d'agir sur les hommes, de les dissuader d'adopter certains comportements vis-à-vis de leur espace forestier et de se rendre autonomes par rapport à d'autres forces telles que celle de l'Etat, symbolisée ici par le service forestier. Pour montrer en quoi ce pouvoir de dissuasion existe et peut même être utilisé contre l'appareil d'Etat, le président de l'association des chasseurs disait ceci :

"Aucun agent forestier n'a jamais encore osé faire un contrôle chez nous. Chaque fois que les agents forestiers rentrent dans notre secteur, ils ne dépassent pas les limites du village de Ouoronina ».

La chefferie entend ainsi garder ses pouvoirs de gestion sur les ressources naturelles. A défaut de pouvoir lutter ouvertement contre la puissance de l'Etat, elle avance ses pouvoirs mystiques pour dissuader les pou- 
voirs publics de la déposséder de ses prérogatives en matière de gestion. De ce point de vue, l'usage de la force occulte que détiennent ces devins et autorités spirituelles est considéré comme essentiel par le personnel politique (Amselle, $1978:$ 351). Il y a là comme une forme de résistance passive face aux changements qui se profilent par rapport aux pouvoirs de gestion attribués théoriquement aux autorités communales élues, en même temps que survient une affirmation de l'autochtonie. Le chef de village disait :

"Ici à Ticko, nous avons toujours été nous-mêmes. Nous n'avons pas changé, nous gardons toujours nos valeurs anciennes. C'est pourquoi nous avons pu conserver nos ressources forestières, c'est pourquoi nous sommes difficiles à vaincre ».

Ce discours évoque l'efficacité de l'autochtonie dans la gestion des ressources. Mais il dénote en même temps d'une certaine frilosité par rapport à d'autres forces susceptibles de supplanter le pouvoir autochtone sur ces ressources. Notamment, une gestion démocratique des ressources, qui semble être le plus grand danger pour leur pérennité.

\section{Néo-traditionalisme, autochtonie et gestion des ressources forestières}

En comparant les deux villages, nous pouvons dire qu'il existe à Ouoronina un système de gestion néo-traditionnel qui essaie de combiner tradition et modernité tout en ne heurtant pas trop l'autorité étatique et en adhérant à une exploitation marchande des ressources. Le domaine forestier relève de l'incontrôlé et par conséquent les autorités villageoises ne peuvent pas s'opposer aux coupes officielles, d'où la tolérance forcée qu'elles observent quant aux agissements des détenus sur la ressource.

Nous constatons, à l'instar de feu professeur Kassibo (2006b), une redynamisation des associations traditionnelles sous l'influence de I'intervention du Projet de Gestion des Ressources Naturelles qui avait mis en place les CVGRN. Ces comités auraient du théoriquement être gérés par les villageois eux-mêmes mais, en réalité, leurs principes de fonctionnement avaient été élaborés par les seules instances de direction du projet. Ces comités ne se sont pas multipliés, parce que fondamentalement dépourvus d'enracinement dans la réalité. Bien que reposant sur des prin- 
cipes " démocratiques " dans leur mise en place, ils fonctionnaient sur des principes gérontocratiques ${ }^{4}$. Ils ont donc été délibérément désaffectés après la fin du projet pour laisser la place aux institutions (karé et donso ton) dans lesquelles se reconnaissaient les populations. En fait, c'est le rétablissement du pouvoir des autorités coutumières sur les ressources qui s'est ainsi opéré.

Les autorités coutumières des deux villages ont des comportements différents si l'on considère les droits en matière de gestion des ressources naturelles mentionnés par Barrière (1996), à savoir les droits de passage, de prélèvement, d'exploitation, d'exclusion et de protection. A Ouoronina, les droits de passage sont reconnus aux pasteurs transhumants, de même que les droits de parcours et de gîte ainsi que ceux de l'accès aux ressources et à leur usage. Les troupeaux peuvent accéder aux zones de pâturage et y séjourner pendant la saison sèche. Il existe cependant des restrictions quant au droit de prélèvement. Le fourrage foliaire constitue ainsi un point de litige entre les organisations locales de gestion et les transhumants. Mais cette contradiction ne s'est jamais aiguisée au point de rejeter les pasteurs et leurs troupeaux hors des pâturages. Les populations considèrent les ressources forestières comme un bien économique qu'il faut utiliser pour satisfaire les besoins. De ce fait, les autorités coutumières ne s'opposent pas à l'exploitation économique de ressources telles que le bois mais elles luttent plutôt pour la réserver à leur propre communauté. Par rapport à cette ressource en particulier, cela conduit à l'exclusion des étrangers, au contrôle de l'espace et au monopole de l'exploitation. Le droit de protection de toutes les espèces est aussi affirmé dans l'interdiction de la coupe de certaines essences particulières ou de bois vert destiné à la vente.

Ainsi la revalorisation des institutions traditionnelles permet, dans une certaine mesure, de faire face aux besoins pressants. Le néotraditionalisme, pour nous, est l'utilisation des pratiques du passé pour gérer le présent. Ainsi est-il donné aux kari et donso ton des rôles (surveillance et mise en défens de l'espace forestier) que pourrait exercer l'ensemble de la société civile. Dans cette optique, les autorités tradition-

4 Pour une expérience similaire au Sénégal, lire Boutinot et Diouf, 2006. 
nelles essayent d'allier objectifs économiques et objectifs de protection et de conservation dans un cadre négocié entre les différents acteurs. Elles ne s'opposent pas frontalement à la gestion de l'Etat. La réglementation est respectée quant aux dessouchages. Le domaine orienté du hameau de Nialafoura échappe à leur contrôle et c'est la gestion de l'Etat qui prévaut toujours à travers l'ancienne Stratégie Energie Domestique, aujourd'hui relayée par l'AMADER (Agence Malienne pour le Développement de I'Energie Domestique et de l'Electrification Rurale). On constate par contre une démarcation par rapport à l'Etat dans l'instauration de taxes illicites servant à financer les charges communes du village et une autonomisation de ce dernier par rapport au service forestier.

A Ticko, les droits cités plus haut sont, quant à eux, tous exclusifs et réservés. Aucun droit de parcours ni de gîte n'est reconnu aux pasteurs transhumants. Le droit de prélèvement appartient aux seuls autochtones, pour les besoins familiaux et de viatique. Le droit d'exploitation économique est totalement nié. Aucun produit forestier ne peut être vendu. Seuls les droits d'exclusion, de protection et d'exercice de la police forestière - sans se référer à l'Etat - prévalent pour les autorités coutumières de ce village. Nous sommes là devant une véritable logique de conservation dont le fondement idéologique est l'autochtonie, c'est-à-dire une justification de l'antériorité de l'occupation de l'espace où la référence à des mythes fondateurs légitime un pouvoir de gestion sur les ressources en excluant les autres acteurs.

Le recours aux institutions traditionnelles, notamment, à la religion traditionnelle dans la gestion des ressources naturelles a été abordé dans la littérature sur la gestion des ressources naturelles. MOGBA (1999) a trouvé que l'un des facteurs invariants encore perceptibles dans les sociétés forestières du Sud-Cameroun est la permanence historique et idéologique du sacré dans le système de représentation sociale lorsqu'on aborde les problématiques de la déforestation de la perte de la biodiversité et de leur mauvaise gestion. Selon lui, cette permanence historique et idéologique est identifiable à travers les discours des vieux valorisant l'existence jadis des gardiens de la tradition et de la forêt. DJAH (2009) a montré comment les acteurs de la religion traditionnelle Ehotilé ont contribué à préserver des îlots forestiers. Ils ont été à l'origine du processus, unique en Côte 
d'Ivoire, qui a abouti au classement de quelques îles de la lagune Aby en parc national.

Cette façon d'intégrer la problématique de la conservation est une construction sociale de l'environnement (Rodary et Castellanet, $2003: 7$ ) qui prend ses racines dans la gestion du passé mais semble viser à se prémunir contre les changements qui s'annoncent pour le futur.

\section{La gestion forestière comme enjeu communal}

Dès la mise en place des collectivités locales, en mai 1999, les autorités communales de Bancoumana ont placé la gestion des ressources naturelles de la commune dans le vaste programme de l'intercommunalité mis en œuvre par l'Amicale des Municipalités du Mandé (AMUMA), dont 16 communes sont membres. La Mairie a été appuyée par un projet de la coopération allemande, le Programme d'Appui aux Collectivités Territoriales de la GTZ (PACT-GTZ). Il s'agissait d'élaborer un plan d'aménagement pour tout le Mandé ainsi qu'une convention locale de gestion des ressources naturelles. La commune a proposé, dans son plan de développement, de mettre en défens les forêts et de lutter contre l'exploitation abusive des arbres et les feux de brousse. La formation des citoyens à la connaissance des textes de gestion des ressources naturelles (code forestier, code domanial et foncier, etc...) et leur traduction en langue malinké figuraient parmi les priorités, ainsi que la mise en place d'un cadre de concertation dénommé "comité de médiation intercommunal ", chargé d'initier un plaidoyer pour le reversement des taxes perçues par les services des Eaux et Forêts aux communes, par le biais de l'AMUMA.

Pour la réalisation de ce programme, le PACT-GTZ a été un partenaire extérieur privilégié, de même que le Mouvement culturaliste N'Ko, d'inspiration mandingue, dont les leaders devaient être, de par leur connaissance du terroir, à même d'exhumer les modes d'administration et de gestion des ressources naturelles (terre, eau, forêt) qui prévalaient dans l'Empire du Mali. Ceux-ci devaient constituer la base d'une gestion communale des ressources conforme aux traditions, aux valeurs et à la civilisation mandingue. 
La première mesure adoptée par les autorités communales en matière de gestion forestière a été le rejet du modèle étatique de la Stratégie Energie Domestique (SED), basé sur l'exploitation rationnelle des ressources par l'aménagement des forêts et la mise en place formelle de marchés ruraux de bois (loi 95 - 003 du 18 janvier 1995 portant organisation de l'exploitation, du transport et du commerce du bois) gérés par des entités associatives dites structures rurales de gestion (SRG). II s'en est suivi l'interdiction totale, depuis l'année 2000, de l'exploitation commerciale du bois-énergie et du charbon de bois dans la commune. Cette décision a fait suite à une convention intra-communale établie entre les différents villages. Il ne peut être fait dérogation à cette règle qu'avec l'accord du Maire qui a l'autorité pour contraindre le service forestier - basé à Siby (chef-lieu de résidence du sous-préfet représentant de l'Etat) - à s'y soumettre. En outre une convention intercommunale a été élaborée avec la commune de Karan contre l'exploitation frauduleuse de la forêt.

Le conseil communal, pour asseoir sa prise de position, a eu recours à la société civile. Ainsi, le Mouvement culturaliste N'Ko a-t-il été sollicité pour sensibiliser les populations à conserver les schémas précoloniaux de gestion des ressources naturelles. Par ailleurs, le Mouvement N'Ko a eu un tel écho favorable auprès des autorités politiques qu'il a été mis à contribution pour faire admettre l'idée de la décentralisation auprès des populations, cette réforme étant le projet politique majeur du premier quinquennat (1992-1997) du Président Alpha Oumar Konaré. Le Mouvement N’Ko s'est donc évertué à démontrer que la décentralisation n'était pas étrangère à la tradition politique malienne. Selon ses idéologues :

"La Constitution décentralisée de l'empire du Mali telle qu'elle a été édictée par Sunjata lors de l'assemblée du Kurugan Fuga est observable dans le fonctionnement actuel des communautés villageoises, de sorte qu'il convient de faire ressurgir ce type d'organisation politique en ramenant, selon leur propre expression, le pouvoir à la maison (ka mara la segin so) » (Kassibo, 2006).

Les autorités communales de Bancoumana se sont alors trouvées confortées dans leur démarche.

En décembre 2003, une conférence de deux jours a été organisée dans le village de Kéniéroba. Les chefs traditionnels de tous les villages de la commune étaient invités. Les conférenciers du Mouvement N'Ko ont fait 
état de leur conception précoloniale de la gestion des ressources naturelles. Selon eux, la forêt en tant que telle ne constituait pas une entité à part dans le Mandé historique : elle était toujours liée à la terre et prise en compte avec elle; ce qui importait dans la culture mandingue, c'était plus la terre que ce qu'elle portait; toutes les terres appartenaient à des lignages. A ce titre, les ressources forestières qu'elles recelaient appartenaient donc à ces mêmes lignages, tout en étant considérées comme propriété commune (bèjè en langue mandingue).

Les nouvelles dispositions législatives (l'ordonnance 00-0279-RP du 22 mars 2000 portant Code domanial et foncier) de la gestion domaniale ont aussi été sévèrement critiquées par les différents orateurs. Ils ont invité les chefs de village à garder leur pouvoir traditionnel de gestion sur les ressources et à résister aux assauts des "gens de Bamako " sensés vouloir faire main basse sur leurs terres et, partant, sur les ressources. On a alors constaté un alignement des nouvelles autorités communales sur ces positions conservatrices relatives à la gestion des ressources forestières par les autorités coutumières.

Pour les conférenciers du Mouvement N'Ko, la forêt était un bien commun accessible à toute la communauté villageoise pour la jouissance des droits d'usage. Pendant que l'exploitation de la terre était sujette à des démarches auprès des chefs coutumiers, l'exploitation forestière était libre dans la mesure où elle ne consistait qu'à satisfaire les besoins domestiques. L'insistance sur la valeur non marchande de la terre et de tous les éléments qu'elle recèle, en particulier les espèces végétales, a été fort marquante. Le représentant du village de Ticko, particulièrement véhément dans ses propos, a ainsi affirmé : "Nous ne laisserons jamais les étrangers annexer nos terres et détruire nos forêts ". Le mouvement culturaliste N'Ko prônait la formalisation des coutumes par des textes écrits notamment dans la graphie du N'ko5. Ce mouvement contribuait ainsi à l'exhumation de ce que ses adhérents appellaient les "valeurs du passé ». Mais à y regarder de plus près, " il apporte une contribution de poids à la

5 Le n'ko est le nom d'une écriture conçue par le Guinéen Solomana Kante en 1949 comme système de transcription des langues mandingues en Afrique occidentale. Des associations N'ko se sont formées autour de promotion de cette écriture et la défense de la culture mandingue. 
définition de l'idéologie des dominants (Amselle, 2001 : 170) ». Cette idéologie, au niveau local, ne pouvait être que le renforcement du pouvoir des lignages dominants au détriment du renforcement d'une société civile non inféodée à la chefferie villageoise et de l'instauration d'une vraie démocratie locale.

Ce discours a émergé sur notre site de recherche au niveau des élites traditionnelles qui ne sont toujours pas d'accord avec l'application de la loi en matière de gestion foncière. II s'agit ici d'un néo-traditionalisme qui essaie de réinventer une sorte d'autochtonie précoloniale qui serait la solution à tous les problèmes de développement et, singulièrement, à la gestion des conflits autour des ressources naturelles.

L'on peut constater sur ce plan, que la position des autorités communales rejoint celle des autorités traditionnelles de Ticko. Pourquoi une telle coïncidence alors que la commune est censée être le lieu de l'affirmation des institutions démocratiques ? Nous pensons qu'une telle prise de position n'est pas sans lien avec une certaine forme de clientélisme politique car, dans le Mandé, l'influence des autorités traditionnelles sur le pouvoir est très forte et les enjeux autour des ressources naturelles sont tout aussi stratégiques. Le candidat qui voudrait se faire élire ou réélire Maire doit se ranger sur les positions de la chefferie. Tel ne fut pas le cas du premier Maire qui n'a pas été réélu parce qu'il avait procédé à des expropriations de terre au profit de lotissements viabilisés dans le chef-lieu de commune. Les autorités traditionnelles de Bancoumana ont mené une vaste campagne de dénigrement contre lui. Elles continuent ainsi d'assurer une gestion traditionnelle des terres, laissant à la Mairie les droits d'enregistrement des transactions foncières. Or il existe un nombre important de villages de la commune où les considérations sont similaires à celles de Ticko en matière de gestion des ressources naturelles. Les politiciens ne prennent donc pas le risque de les contredire et cela au détriment de l'affirmation de l'autorité des institutions démocratiques.

Pourtant, ces compétences de gestion n'ont pas été transférées par l'Etat aux collectivités locales. Mais quand bien même ce serait le cas, se poserait le problème de la mise en œuvre pratique sur le terrain. Les villages étant les lieux d'exercice de l'autorité traditionnelle, comment cette dernière pourrait-elle se dessaisir de ses pouvoirs de gestion au profit d'une gestion communale ? Tel semble être l'enjeu fondamental autour de 
la gestion de ces ressources. Cela a déjà pu être constaté à travers l'exemple de la gestion des carrières, qui a été retirée aux villageois pour être insérée dans les prérogatives de la commune : la Mairie a fixé des taxes de redevance dont le recouvrement s'est avéré difficile parce qu'elle n'avait pas d'emprise directe sur l'exploitation.

Les conflits autour des ressources peuvent surgir à tout moment entre les différents acteurs. C'est pourquoi l'AMUMA, avec l'appui du PACT-GTZ, a élaboré une convention locale de gestion durable des ressources agropastorales de neuf communes du cercle de Kati, dont la commune de Bancoumana. Selon cette convention, la prévention et la gestion des conflits passent par un mécanisme ascendant qui va de la base au sommet. Tout conflit surgissant ou en voie de l'être est porté devant un conseil des anciens au niveau du village. Si aucun règlement n'est obtenu à ce niveau, il faut recourir aux autorités communales. Quand celles-ci n'arrivent pas à trouver la solution, le différend est renvoyé devant l'AMUMA. Dans le cas où toutes ces voies de recours sont épuisées, les protagonistes doivent s'adresser à la justice. La grande hétérogénéité des acteurs dont les intérêts sont souvent divergents permettra-t-elle une mise en pratique de cette convention?

L'application de certaines de ses clauses constitue en soi des problèmes. Il est ainsi dit dans l'article 37 que l'exploitation du bois et du charbon à des fins commerciales est totalement interdite sur toute l'étendue des neuf communes. En son article 38, la mutilation des arbres pour nourrir les animaux est aussi totalement interdite. Or les conditions d'utilisation et d'exploitation des ressources sont fixées par la loi et ces dispositions sont tout à fait contraires à la politique de l'Etat concernant l'exploitation des ressources forestières, notamment la gestion rationnelle par l'aménagement des forêts et la création des marchés ruraux de bois, dont nous avons signalé plus haut l'existence à Kéniéroba et dans la plupart des communes concernées.

Les carences de l'Etat, tant du point de vue juridique qu'en termes de régulation sociale, ont amené les différents acteurs à élaborer des conventions locales de gestion des ressources naturelles. Ces conventions sont établies entre les populations sous l'égide des responsables communaux et des Organisations Non Gouvernementales (ONG) ou des programmes bilatéraux d'appui mais leur application n'est pas aisée sans le recours à l'Etat. 
La plupart des conventions locales, dont celle élaborée par l'AMUMA, souffrent en effet de leur non respect. Cette difficulté de mise en œuvre se pose du fait d'un manque de maîtrise des différentes clauses par les usagers, de la faiblesse de la diffusion des conventions à l'échelle locale et de la non fonctionnalité des mécanismes de concertation entre les organes villageois (Dicko, $2002: 23)^{6}$.

Deux thèses s'affrontent quant aux conventions locales. Le cadre légal, selon les partisans de la légalité des conventions locales, est renforcé par la politique forestière nationale qui souligne que celle-ci « vise à déléguer de façon durable la gestion des ressources sur des espaces bien définis aux entités villageoises qui en feraient la demande " (MDRE, 1995 : 29). Pour eux, l'élaboration des conventions locales est parfaitement autorisée par les textes. Les tenants de la seconde thèse ne contestent pas la légalité du principe de la convention mais soulignent plutôt le caractère illégal de certaines dispositions des conventions locales ou l'absence de fondement juridique de certaines pratiques (Djiré, 2004 : 8-9).

La commune de Bancoumana, à travers cette convention, est dans une logique de préservation des ressources forestières mais il faut aussi s'interroger sur les dispositifs législatifs et réglementaires permettant l'application d'une telle convention. Tous les acteurs accepteront-ils de suivre la voie indiquée par la convention ? Le Maire lui-même ne reconnaît-il pas que, de plus en plus, le bois et le charbon de bois sont exploités dans le village de Niakanabougou ? Des processus discriminatoires se dessinent. Ils visent l'appropriation des ressources par certaines communautés villageoises (Mbodj, 2005) et l'exclusion ou la mise à l'écart des communautés considérées comme étrangères aux terroirs et voulant profiter des ressources. On voit là toutes les difficultés d'application des conventions locales.

\section{Conclusion}

La gestion démocratique des ressources forestières dans un cadre décentralisé est confrontée aux idéologies du néo-traditionalisme et de

$6 \mathrm{Cf}$. le texte de Antona et al. dans ce même numéro. 
l'autochtonie qui se traduisent par l'exclusivité des droits et l'exclusion de certaines communautés. Le recours au néo-traditionalisme et la référence au mythe fondateur de l'autochtonie constituent des instruments de légitimation prioritaires permettant à certains groupes de maintenir leur droit et leur contrôle sur les ressources. Ils servent de justificatifs pour asseoir davantage le pouvoir de l'autorité locale dans la gestion des ressources forestières. La compétition autour des ressources constitue ainsi l'une des conditions de la montée des revendications identitaires dans le champ social. Mais n'est-ce pas une façon de retarder la gestion démocratique des ressources?

Les évolutions dans le secteur forestier, il y a un peu moins d'une décennie, ont été marquée par un un échec patent de la gestion participative et la recherche d'un équilibre de pouvoir de gestion des ressources entre des acteurs hétérogènes. Au lieu de corriger les insuffisances de la gestion démocratique déjà engagée, l'Etat a préféré une "recentralisation " par l'adoption le 12 juillet 2012 d'une nouvelle loi qui supprime les trois types d'exploitation et qui récupère le discours international à travers la Convention africaine de Maputo sur la nature et les ressources naturelles et les grands principes de la Conférence Rio (1992) sur l'environnement et le développement. Malgré ce changement institutionnel, la question du pouvoir de différents acteurs sur les ressources forestière n'est pas tranchée. Comment les relations de pouvoir vont-elles se jouer entre les entités communales et les entités villageoises où le néotraditionnalisme et l'autochtonie montent en force à la faveur de la démocratisation politique ? Tels semblent être les nouveaux enjeux qui se dessinent dans la gestion démocratique des ressources. Une délégation de pouvoir de gestion à des instances élues pourrait être une issue favorable dans le cadre d'une gestion concertée des ressources forestières au niveau local.

\section{Bibliographie}

AMSELLE, J-L., 1978, "La conscience paysanne : la révolte de Ouolossebougou (juin 1968, Mali) ", Revue canadienne des Etudes africaines, vol. 12, n³ : 339355. 
Seydou Keita

AMSELLE, J-L., 2001, Branchements. Anthropologie de l'universalité des cultures, Paris, Flammarion, 265 pages.

BARRIERE, O., 1996, Gestion des ressources renouvelables et conservation des écosystèmes au Sahel: le foncier-environnement, Thèse de doctorat en droit de l'Université Paris I, tome 1, $133 \mathrm{p}$.

BOUTINOT, L. et DIOUF, C.N., 2006, Quand certaines approches participatives engendrent des formes ambiguës de mobilisation de la société civile. Quelques exemples à propos de la gestion des ressources forestières au Sénégal, In : Bertrand A., Karsenty A., Montagne P., (Eds) L'Etat et la gestion locale durable des forêts en Afrique francophone et à Madagascar, Paris, Cirad- L'Harmattan : 195-212.

CAMARA, S., 2002, "La décentralisation au Manden : cas des communes rurales de Bankumana, Minijan, Nyagadina et de la commune urbaine de Karan » in Pouvoirs locaux, pouvoirs d'Etat, démocratie et décentralisation au Mali Rapport final, Bamako, ISH - EHESS - MAE, 423 p.

DJAH, F-M., 2009, "Religion traditionnelle et gestion durable des ressources floristiques en Côte d'Ivoire : Le cas des Ehotilé, riverains du Parc National des îles Ehotilé ", VertigO - la revue électronique en sciences de l'environnement [En ligne], Volume 9 Numéro 2 | septembre 2009, mis en ligne le 22 septembre 2009, consulté le 04 janvier 2014. URL: http://vertigo.revues.org/8661, DOI : 10.4000/vertigo.8661.

DIALLO, Y., 1995, Les Peuls, les Sénoufo et l'État au nord de la Côte d'Ivoire. Problèmes fonciers et gestion du pastoralisme, Bulletin de l'APAD [en ligne], $\mathrm{n}^{\circ} 10,1995$, mis en ligne le 05 juillet 2007, consulté le 10 avril 2014. URL : http://apad.revues.org/1131.

DICKO, A.K., 2002, Les conventions locales dans la gestion des ressources naturelles au Mali, Bamako, PACT-GRN, Etudes n¹4, 44 p.

DJIRE, M, 2004, Les conventions locales au Mali : une grande nébuleuse juridique et un pragmatisme en GRN, Dakar, IIED Sahel, $89 \mathrm{p}$.

Direction Nationale des Ressources Forestières Fauniques et Halieutiques, Direction Nationale de l'Hydraulique et de l'Energie, 1993. Stratégie pour l'Energie Domestique : Plan d'action détaillé, Bamako.

KASSIBO, B, 2006a, "Mali : une décentralisation à double vitesse? Ka mara la segi so ou le lent et délicat retour du pouvoir à la maison ", in Fay, C., Koné, Y.F. et Quiminal, C. (eds) Décentralisation et pouvoirs en Afrique. En contrepoint, 
modèles territoriaux français, Paris, IRD Editions, coll. Colloques et séminaires : $67-95$.

KASSIBO, B, 2006b, Approches participatives et gestion décentralisée de la forêt du Samori dans la commune de Baye, région de Mopti (Mali) in Afrique et Développement, Vol. XXXI, No. 2, 2006, pp. 215-242

LEYNAUD, E., et CISSE, Y., 1968, Paysans Malinké du Haut Niger (Tradition et développement rural en Afrique Soudanaise), Bamako, Editions Populaires, $451 \mathrm{p}$.

Ministère du Développement Rural et de l'Environnement, 1995, La Politique Nationale Forestière, Bamako.

MOGBA, Z.,1999, Etude des systèmes locaux de gestion des ressources forestières à Djoum (Cameroun), Central African Regional Program for Environnement (CARPE), 42 p.

MBODJ, B.F., 2005, Gestion décentralisée des ressources forestières: enjeux économiques et sociaux-spatiaux. L'exemple de la commune rurale de Siby, Mémoire de Maîtrise, Université de Paris I, Pantéon-Sorbonne, 120 p.

RODARY, E. et CASTELLANET C., 2003, "Les trios temps de la conservation » in Rodary E., Castellanet C. \& Rossi G. (dir.) Conservation de la nature et développement, Paris, Karthala : 5-44.

SAWADOGO, A.R., 2001, L'Etat africain face à la décentralisation, Paris, Karthala, $278 \mathrm{p}$.

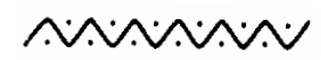

Seydou Keita est Professeur d'Anthropologie à l'Institut National de Formation des Travailleurs Sociaux de Bamako (Mali)

E-mail : seydouster@gmail.com 